\title{
Rare Case of Pelvic Peritonitis Misdiagnosed by Ovarian Malignancy, Referred Following Chemotherapy
}

\author{
Maliheh Arab, ${ }^{1,2, *}$ Adele Ashoori Barmchi, ${ }^{1}$ Seyedeh Neda Kazemi, ${ }^{1}$ and Atoosa \\ Gharib $^{3}$ \\ ${ }^{1}$ Preventative Gynecology Research Center, Imam Hossein Medical Center, Shahid Beheshti University of Medical Sciences, Tehran, IR Iran \\ ${ }^{2}$ School of Medical Education Sciences, Shahid Beheshti University of Medical Sciences, Tehran, IR Iran \\ ${ }^{3}$ Department of Pathology, Shahid Beheshti University of Medical Sciences, Tehran, IR Iran \\ ${ }^{*}$ Corresponding author: Maliheh Arab, Preventative Gynecology Research Center, Imam Hossein Medical Center, Shahid Beheshti University of Medical \\ Sciences, Tehran, IR Iran. Fax: +98-2177543634, E-mail: drmarab@yahoo.com \\ Received: April 7, 2014; Accepted: May 1, 2014
}

\begin{abstract}
Background: Tuberculosis is an old disease that is still one of the main causes of death in the world. Nearly 60-67\% of TB patients are from developing countries. Proper treatment results resolution in almost all cases. Pelvic TB occurs in about 10\% of pulmonary TB patients. The present case reviews a TB case misdiagnosed in a young woman with a pelvic mass and ascetic fluid.

Case Report: A35-year-old woman living in an area exposed to immigrants from TB endemic regions presented with ascetic fluid and a pelvic mass and high titer of sacrum CANS (350). She was misdiagnosed with ovarian malignancy due to a positive malignancy report of ascetic fluid. After chemotherapy she was referred for definite operation. In the surgical field, a TB diagnosis was made.

Conclusions: Review of preoperative reports and careful history of the case revealed that had an awareness about TB existed in this case, a proper diagnosis might have been made before the erroneous chemotherapy. She was young, suffering from pelvic pain, fever, and weight loss coming from an exposed area to TB cytology of ascetic fluid included dominancy of lymphocytes. Relying on a positive cytology finding for malignancy might be questionable.
\end{abstract}

Keywords: Tuberculosis; Pelvic Inflammatory Disease; Ovarian Neoplasms

\section{Background}

Tuberculosis (TB) is an ancient and well-known disease that still continues to be among the main causes of death all over the world. Mycobacterium tuberculosis is the responsible bacteria and involves the lungs in most cases and other organs, as well. In a 2009 World Health Organization (WHO) report, nearly 5.8 new tuberculosis cases are reported. Ninthly-five percent of these reported cases occurred in developing countries. These reported cases are expected to comprise $60-67 \%$ of true cases. WHO (2009) estimates that there are 9.4 million tuberculosis cases in the world, with most cases from Asia, Africa, the Middle East and Latin America(1).

Proper treatment results in nearly a complete cure for all patients. Without proper treatment, however, death might occur in more than half of involved people (1).

Tuberculosis can involve every vital organ in the body including bone, meanings, kidney, epididymis, fallopian tube and other sites. Female genital tuberculosis is rare, although TB is a health problem worldwide (2). Regarding the female reproductive tract, the most common route of infection is thru hematogen followed by the lymphatic pathway (3). Pelvic tuberculosis might be considered due to clinical presentation. Gynecologists, however, are seldom focused on this as it is a rare presentation. In most cases, diagnosis is retarded because of vague presenta-

tion. Howard Kelly, once said that when competent gynecologists disagree about the diagnosis of an obscure pelvic condition, it usually is diagnosed as either an "old ectopic pregnancy or pelvic tuberculosis"(4).

This case reports on TB misdiagnosed as an ovarian malignancy.

\section{Case Report}

That was a 35-year-old multiparous woman with history of four births and cesarean operations, with the last cesarean 7 months before admission. She was from Zanjan city located in the northwest of Iran. She resided in Qom which has immigrants from Afghanistan where there is a high incidence of tuberculosis. The patient presented with a frequent productive cough a few weeks after the cesarean, which was considered as mild postoperative respiratory symptoms by local physicians. Her symptoms were followed by weight loss, night fever, abdominal pain and progression to abdominal distention. Four months later she was visited again and her abdominal sonography revealed ascitis with a normal uterus and ovaries. Endoscopy and biopsy just showed patchy erythematic in gastric ant rum. Repeat sonography by a more expert person clarified a solid modularity in the peritoneal cavity and probable metastatic lymph nodes, as there were several soft tissue

Copyright (C2015, Iranian Society of Clinical Oncology. This is an open-access article distributed under the terms of the Creative Commons Attribution-NonCommercial 4.0 International License (http://creativecommons.org/licenses/by-nc/4.0/) which permits copy and redistribute the material just in noncommercial usages, provided the original work is properly cited. 
masses each about $3.5 \mathrm{~cm}$ in the anterior abdominal wall below the umbilicus beside ascitis. Abdominal and pelvic CT scan was in favor of a $9 \times 3 \mathrm{~cm}$ mass in anterior inferior abdominal along with several foci of fluid-filled cavities in a subcutaneous area with congerted peritoneum and ascitis. Pulmonary CT scan report was normal. Positive laboratory finding was serum CA125 titer of 380 IU. A test of aspiration of peritoneal fluid revealed 58\% lymphocytes in cellularity and positive for malignancy cells. Systemic chemotherapy targeting ovarian cancer was done five times. After chemotherapy courses, repeat se CA125 titer was 32 and a significant reduction of ascitis was reported in an abdominal CT Scan. A low abdominal mass close to the abdominal wall of about $9 \mathrm{~cm}$ was persistent besides peritoneal seeding and located in a fluid collection in the abdominal wall and peritoneal cavity.

The patient was referred to this gynecology center. On admission, vital signs were normal with a temperature of $37^{\circ} \mathrm{C}$. After a review of documents, a physical exam palpation of the mass close to a thick abdominal wall was considered as a severe adhesion. A repeat abdominal-pelvic sonography was in favor of a septate mass with high vascularity. In color Doppler, near the left ovary there was another smaller mass in posterior cul-de-sac and loculations in subcutaneous tissue of the abdominal wall. These findings suggested neoplastic lesions of the ovary. Free peritoneal fluid was scant. Due to atypical findings, the patient was presented in a joint clinic of a multi-disciplinary decision making session. She was scheduled for laparotomy and staging with stand-by frozen section. Laparotomy was done about seven months after cesarean. Operative findings revealed multiple micro abscess in a subcutaneous area measuring 1-4 centimeters. All were resected. Extensive adhesion of the small and large intestine and inflamed and thick omentum in the pelvic cavity was dissected.

A cystic mass of about 6-7 cm was revealed in the left side position of a cul-de-sac adherent to the recto sigmoid. In was very hard to dissect, so aspiration was done and a yellowish colored fluid was extracted resulting in the possibility of cyst wall resection for frozen section. The results of the preliminary operation pathology consult was necrotizing granulomatous infection strongly in favor of tuberculosis. The operation was finished in this step. The permanent pathology report confirmed the preliminary operation suggestion. A four-drug anti-tuberculosis treatment was started by the infectious disease group of the hospital. There was no complication during the post operation period.
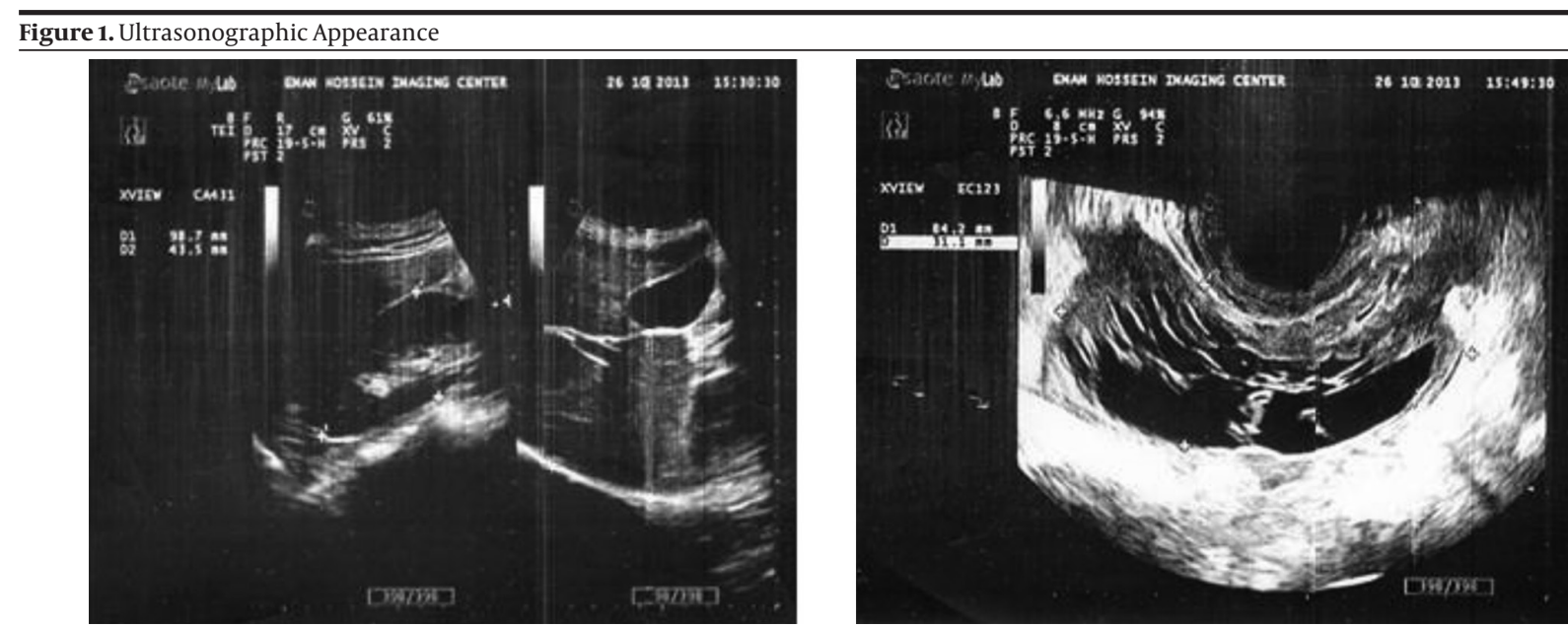

A \& B, cystic lesion with septation in post culdesac and near left ovary is found.

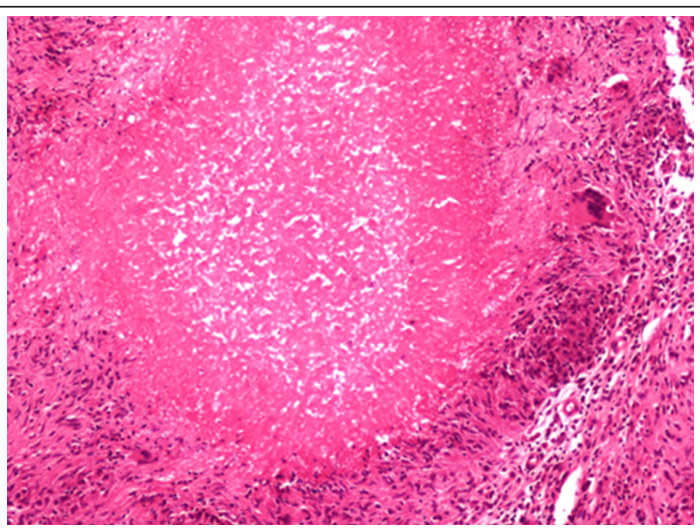

Figure 2. Abdominal Wall View; Multiple Subcutaneous Abscesses

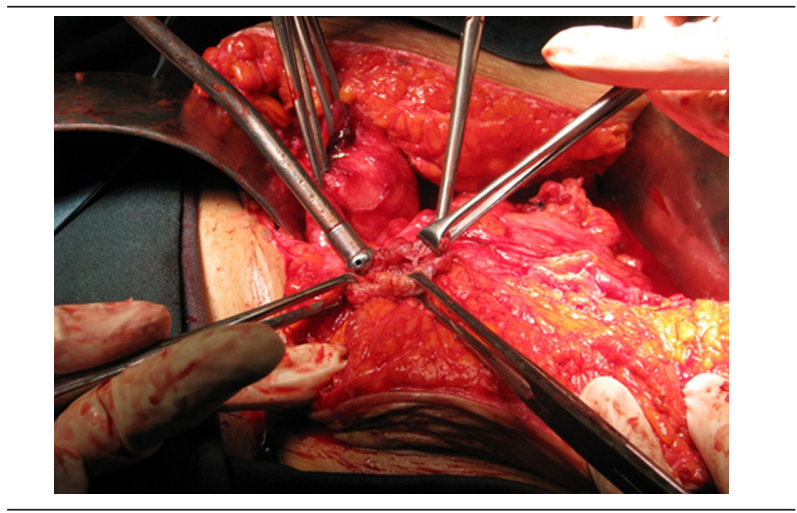

Figure 3. Histopathologic Feature; Fibrous Tissue With Necrotizing Granulomatous Inflammation and Foci of Abscess Formation 


\section{Discussion}

Tuberculosis infections are again increasing and consideration is necessary, especially in endemic areas such as Asia and in HIV-positive cases. Although genital tuberculosis is rare, every gynecologist should pay attention to atypical presentations of the disease.

Final diagnosis is made by pathology in most cases. Sometimes, mucosal proliferation of the fallopian tube due to tuberculosis might simulate primary carcinoma of the tube. In pathologic examination, tuberculosis peritonitis is mostly associated with pelvic disease and presents in two forms including wet and dry (adhesive). In adhesive (dry) type, bowel injury is probable in the operation field due to the adhesions. In wet type, free fluid exists in the peritoneal cavity. The peritoneal surface is covered by several small tuberculosis. Tuberculosis peritonitis and carcinomatous peritonitis are in the differential diagnosis of each other with elevated se CA125 level making the diagnosis more difficult (5).

Pelvic tuberculosis occurs mostly in the age group from $20-40$ years old. The most common clinical presentations of pelvic tuberculosis includes pelvic pain, general malaise, menstrual irregularity and infertility. All the cases of pelvic tuberculosis in exam show tenderness with a low-grade fever present in most cases. About 10-20\% of patients with the history of tuberculosis lung disease, are involved by pelvic disease. Due to the rarity of pelvic tuberculosis, it is not considered in differential diagnosis and a gynecologist may not be aware of it. In some cases, the diagnosis is only made in the laparotomy field (4). If a tuberculosis diagnosis is made, surgical intervention is seldom needed. In the case of pelvic mass and ascitis in wet tuberculous peritonitis, adnexal tenderness, fever, history of pulmonary tuberculosis and infertility are helpful in differentiating TB from ovarian malignancy.

Serum CA125, as a non-specific tumor marker is elevated in many benign and malignant conditions, except for ovarian malignancy (6). In a study median level of se CA125 in ovarian cancer was significantly higher than TB peritonitis $(\mathrm{P} \leq 0.001)$. Just one patient (21\%) in a tuberculosis group had a higher than $2000 \mathrm{U} / \mathrm{ml}$ level of serum CA125 (7). In ascitis fluid of TB cases lymphocytes are prominent and if proper suspicion exists, acid-fast mycobacterium tuberculosis can be identified by the Zeill-Nelson method (8).

The present case had regional exposure to immigrants from an endemic area (Afghanistan), age of 35, and with fever and pain. Se CA125 level was 380IU. Due to ascitis and pelvic mass with no awareness of TB, peritoneal fluid aspiration, although with $68 \%$ lymphocyte predominance, was reported as positive for malignancy and chemotherapy had been prescribed before referral for surgical doubling. Awareness and considering TB in differential diagnosis seems to be the main factor in correct diagnosis. However, making sure of malignancy by histology conformities instead of cytology might prevent such errors. An interesting point in this patient was a redacting of se CA125 and ascitis after chemotherapy. A flare-up of tuberculosis following chemotherapy was not shown.

In conclusion, TB should be considered in ascitis and mass especially in young women (9) exposed to endemic regions. Pelvic pain and tenderness, history of pulmonary $\mathrm{TB}$, infertility and fever are helpful, certainly if clinical awareness exist. Chemotherapy just based on positive cytology can result in errors such as in the present case.

\section{References}

1. Mehta JB, Dutt A, Harvill L, Mathews KM. Epidemiology of extrapulmonary tuberculosis. A comparative analysis with pre-AIDS era. Chest. 1991;99(5):1134-8.

2. Wehner JH, De Bruyne K, Kagawa FT, Campagna AC, Jensen WA, Kirsch CM, et al. Pulmonary tuberculosis, amenorrhea, and a pelvic mass. West J Med. 1994;161(5):515-8.

3. Chavhan GB, Hira P, Rathod K, Zacharia TT, Chawla A, Badhe P, et al. Female genital tuberculosis: hysterosalpingographic appearances. BrJ Radiol. 2004;77(914):164-9.

4. Corapcioglu F, Guvenc BH, Sarper N, Aydogan A, Akansel G, Arisoy ES. Peritoneal tuberculosis with elevated serum CA 125 level mimicking advanced ovarian carcinoma in an adolescent. Turk $J$ Pediatr. 2006;48(1):69-72.

5. Rock JA, Jones HW. Pelvic Inflammatory Disease. In: Seigafuse S, Shaw R, editors. TeLinde's Operative Gyneocology. Lippincott Williams \& Wilkins; 2008. pp. 676-84.

6. Ilmer M, Bergauer F, Friese K, Mylonas I. Genital tuberculosis as the cause of tuboovarian abscess in an immunosuppressed patient. Infect Dis Obstet Gynecol. 2009;2009:745060.

7. Wang D, Zhang JJ, Huang HF, Shen K, Cui QC, Xiang Y. Comparison between peritoneal tuberculosis and primary peritoneal carcinoma: a 16-year, single-center experience. In: Longo D, Fauci A, Hauser S, Kasper D, Jameson J, Loscalzo J, editors. Harrisons Principles of Internal Medicine. McGraw Hill Professional; 2012. pp. 1340-1.

8. Wang D, Zhang JJ, Huang HF, Shen K, Cui QC, Xiang Y. Comparison between peritoneal tuberculosis and primary peritoneal carcinoma: a 16-year, single-center experience. Chin Med J (Engl). 2012;125(18):3256-60.

9. Bae SY, Lee JH, Park JY, Kim DM, Min BH, Rhee PL, et al. Clinical significance of serum CA-125 in Korean females with ascites. Yonsei Med J. 2013;54(5):1241-7. 